\title{
Design and Implementation of Smart Home Voice Control System based on Arduino
}

\author{
Min Liu \\ Dezhou Vocational and Technical College, Dezhou, Shandong, 253034
}

Keywords: Smart Home Voice Control System; implementation; design and research

\begin{abstract}
The main research contents of this paper is designing the overall architecture of the smart home control system. The system is divided into the $\mathrm{H}$ layer, the upper layer which is the communication layer, the middle layer which is the relay control layer, and the first layer is the home system, and the Bluetooth infrared repeater based on Arduino development board infrared device equipped with Bluetooth wireless module; with the analysis of infrared control theory, Bluetooth module technology and Arduino related technologies, according to the overall system architecture design, the system functions are divided into hardware systems and software operation and management of two subsystems for infrared signals; Repeater information and other data sheets were designed and implemented. Star remote control was used as an example. Based on Arduino development, a Bluetooth infrared repeater was designed in conjunction with Bluetooth wireless communication technology, including transmission and reception, and encoding and decoding were implemented with Bluetooth module selection analysis, Bluetooth transmission circuit and serial communication; detailed implementation of the software operation and management part is proposed.
\end{abstract}

\section{Introduction}

The development of today's science and technology is amazing. Various industries have taken this express train. Manufacturing, service, agriculture and other industries have achieved a more efficient, high-quality and high-speed product in this rapid development today, satisfying the material, spiritual, and cultural needs of the broad masses of people. The development of the Internet industry is even more rapid. There has been a major reshuffle of the industry. In this major reshuffle of technology, smart homes have been valued by most technology companies, and they have begun to make achievements in the home industry and make people live in a home environment. Become more intelligent, convenient and comfortable. With the development of the Internet, various household devices have been connected to the Internet. Therefore, the demand for various embedded devices to access the Internet has increased dramatically, which has promoted the generation of smart homes. Smart homes can also be referred to as smart homes and are represented by Smart Home [1]. In simple terms, smart homes connect lighting, home appliances, background music, remote controls, electric curtains, gas warnings, security devices, home theaters, etc. into a single network - the Internet of Things [2]. Utilize the local server namely the gateway to realize the unified management, achieve the data acquisition and the control of the apparatus, bring the convenient, comfortable living environment to the user. Today's intelligent products emerge in an endless stream. There are numerous intelligent products for the home industry, and the household industry has a large number of users. Therefore, the smart home has a lot of room for development. Although major technology companies are also striving to deploy smart home development strategies, and there are already some smart products available, most smart home products on the market today are expensive and generally unacceptable to families. Therefore, it is particularly necessary to develop a smart home product that is inexpensive, fully functional, and capable of long-term stable operation. The core of the smart home system is the design of the gateway. Now most systems are independent of the gateway into a separate device, using a separate PC server or embedded custom gateway service, and then through the home router to achieve the connection of the home device and the Internet. This will make the home network too many devices, wiring 
difficulties, and increased costs.

\section{Arduino Development Technology}

Arduino is a convenient, flexible, and easy-to-use open source electronic prototyping platform, including Arduino microcontroller hardware platform and development integrated environment IDE. It was taught by Italian Massimo Banzi that there was not a good microprocessor because of student complaints, which led him to develop a user-friendly microprocessor hardware platform. So he worked with a hardware engineer and his students to design the Arduino. Later, Banzi, Cuartielles, and Mellis released the design map to the Internet, open-sourced all of their hardware and software, and made its application community huge. Since the development of Arduino, there have been many models and many compatible controllers available. Its hardware is AVR microcontroller, 8-bit and 16-bit, software programming language is similar to $\mathrm{C}$ language, easy to grasp. Common categories include Arduino Uno, Arduino Nano, Arduino mini and more. The number of products developed using Arduino is now numerous, such as robotic arms, 3D printers, quadrotors, etc.

The Arduino platform has many different versions and is used in different scenarios. There is a smaller nano, slightly larger uno, the text uses the "Arduino uno" version as an example, based on the ATmega328 microcontroller board, you can directly select the USB power supply, especially convenient.

\section{System Hardware Design}

The system hardware includes the Wi Fi core board, Arduino control center, USB expansion, and hardware design of each terminal. Among them, the Wi Fi core board, the Arduino control center, and the USB expansion board constitute an intelligent gateway, which enables all devices in the home to connect to the Internet and realize data interaction. The control center adopts Arduino expansion board and connects Wi Fi wireless module to Arduino to realize data collection and downlink control for each terminal.

The USB expansion board is responsible for mounting the $U$ disk in this design, which provides space for the installation of application software. It is a 4-channel expansion chip with FE1.1s as the core chip, ultra-low power consumption and USB 2.0 support for hot-plugging, and is a USB expansion master IC for USB 2.0 with 4 USB expansion ports. It is a chip with STT data architecture with Self Power overcurrent protection function and excellent data interaction function. The chip features are as follows. The USB expansion board was originally designed to add four USB and one TF card interface, as shown in Figure 3.9. Later, when designing the system, only one SD card needs to be mounted to install software such as Python and My SQL. Multiple interfaces, eventually decided to extend only two USB interfaces and a TF card interface, this expansion board can also be used when the reader, used to read the contents of the relevant SD card.

The Arduino expansion board is designed with an ATMEG644PA chip, 64K flash, and two serial ports. Serial is used to communicate with Open Wrt and SPI is used to communicate with Wi-Fi module NRF24L01. ATMEG644PA is a high-performance, low-power AVR microcontroller with an advanced RISC architecture, 131 powerful instruction sets, a single clock cycle execution, 64K bytes of in-system programmable flash capability, and a 10,000 write/erase cycle. The boot code of the selected part is independently locked at the same time as the read/write operation of the system-program-on-chip startup programtrue; $2 \mathrm{~K}$ bytes of EEPROM endurance. In this design, the Anduino expansion board is responsible for controlling the Open Wrt serial communication in the gateway. The data sent from each terminal is processed, then passed to Open Wrt, and then passed to the cloud server by Open Wrt to realize the data exchange with the mobile phone. pass. The Arduino expansion board plays a crucial role in this smart gateway.

Due to the open source nature of the Arduino platform, download the hardware circuit diagram on the Arduino official website, replace the chips in it with what we need, and modify the other peripheral circuits slightly to meet our needs and support Arduino's various library functions and IDEs. Development environment. The Arduino expansion board is based on the official open source 
circuit.

\section{Arduino Control Center Programming}

Arduino as the gateway's control center mainly implements Open Wrt serial communication. If data is present, the data is immediately sent to Open Wrt and Open Wrt saves the data to the database or POST to the cloud server. NRF24L01Wi Fi module communication processing, Wi Fi module is responsible for the data exchange between each terminal want to and gateway.

Arduino platform includes Arduino hardware and integrated development environment IDE. The Arduino is favored by so many people, mainly because it encapsulates a lot of microcontroller registers, timing counters and various functions, and does not need to operate the bottom layer. However, in the actual development, the library function file that Arduino IDE brings is far from enough, so also need to build the library function file by oneself. The NRF24L01Wi Fi module used this time is not found in the built-in library function file, so you need to build your own library. The library file used this time is shown in Figure 4.10, where <SPI.h $>$ mainly encapsulates SPI communication, <Mirf.h>, <n RF24L01> and <Mirf Hardware Spi Driver.h> are all on NRF24L01Wi Fi. Module communication package. For example, NRF24L01 internal register configuration, auto-response, receive address, auto-retransmit, RF channel, register status, send detection and a series of configuration package. In the process of writing the program does not need to operate on the NRF24L01, just call it.

Yeelink is a smart cloud service platform that provides cloud services. With the rapid development of smart devices, the cloud services provided by this platform are favored by developers and provide a large number of sensor data access and cloud storage for the majority of developers. Program. The platform is to allow embedded devices to use the HTTP protocol to implement the transmission of sensor data and provide detailed API development documents for developers to use. In this design, each sensor data, control data, and camera data are saved to the cloud platform via HTTP, providing convenience and reliability for remote monitoring. The user first needs to go to the platform to register. If the registration is successful, an APIKEY will be obtained. After that, all sensor camera devices need the APIKEY to authorize to operate. Each device will have a URL address through which data can be uploaded to the sensor. It should be noted that the format of data transmitted by digital and image-based devices is different. The Yeelink development documentation states that all device data is stored in a key-value format. Different data is sent through different HTTP protocols. Sending a relevant HTTP GET request to the PATH/datapoint address will return relevant data. The key corresponding to the value data point information. When there is no key value, the new data node of the value sensor is returned. There are differences between the data format of the image sensor and the digital sensor but there are similarities. The image information is also composed of key and value, and the difference between the numerical value and the numerical value is that the image information is saved in size, width and height, and the image content is a binary file. Sending an HTTP GET request to the address of PATH/photos will send the user-specified image sensor a jpg, png, gif image. The Yeelink cloud server will add the current time as the key of the image and also upload the image with the permission of the APIKEY of the conference. The upload time must be at least 10 seconds or an error will occur. Under the premise of obtaining the APIKEY authorization, sending the relevant HTTP GET request to the PATH/photo/content address will return the corresponding value of the corresponding key binary image information. When there is no key value, the most recent image information of the secondary image sensor is returned. The above mainly completed the design of the software part of the system, including the installation of My SQL, Python, the programming of Python scripts that interacted with the Arduino and the cloud server, the writing of the entire home control center Arduino software, and the Android client with voice control function. design. At this point, the basic design of the system is completed, and the corresponding tests will be conducted below. 


\section{Conclusion}

The emergence of modern household appliances has provided great convenience for people's families, and it has greatly replaced household work. Accompanying this development is the human-computer interaction technology between humans and household appliances. This article studies the topic of smart home control and aims to strengthen this interaction and achieve friendly control of the home. Under the direction of this research direction, this article applies Bluetooth and infrared control technology in the home control system. Through the infrared signal to learn the control signal of the home equipment, and the home to conduct unified management, use the Arduino development board to produce infrared transmitter; The research of this paper Wang Zuohe's research results include: The overall architecture design of the smart home system, the system is divided For layer $\mathrm{H}$, the top layer is the communication layer. The control host communicates with the outside through the Internet, cable television network, or PSTN. The middle layer is the relay control layer. The control signal is achieved through the switch light weight controller and the Bluetooth infrared repeater. The second floor is the home system, which includes infrared remote control devices and infrared remote control devices. These devices are controlled through switches or intensity controllers; the Bluetooth infrared repeater passes infrared equipment based on the Arduino development board. Equipped with a Bluetooth wireless module to achieve; analysis of the system's key business processes and related theoretical and technical.

\section{References}

[1] Wang Zhen, Yang Xiaolan. Smart Home System Design Based on Android and Arduino[J]. Software Guide, 2014(12):94-96.

[2] Tong Xiaoyi, Fang Bingyi, Zhang Yunyong. Analysis of the development of smart home of Internet of Things[J]. Mobile Communications, 2010(9):16-20.

[3] Jiang Yanliang. Remote control system based on Android smart terminal[J]. Electric Technology and Applications, 2012(8).

[4] Zhang Yun. Windows Android application development environment construction detailed [J]. computer computing generation, 2016 (2): 63.

[5] Qin Hua. Design of environment monitoring system based on Arduino[J]. College of Mechanical Engineering, Chongqing University, 2013 